\title{
Prospective Assessment of Trocar-Specific Morbidity in Laparoscopy
}

\author{
Alessandra Cristaudi • Marie-Laure Matthey-Gié • \\ Nicolas Demartines · Dimitri Christoforidis
}

Published online: 25 July 2014

(C) Société Internationale de Chirurgie 2014

\begin{abstract}
Background The purpose of the present study was to challenge the hypothetical advantage of single port laparoscopy (SPL) over conventional laparoscopy by measuring prospectively the morbidity specifically related to conventional trocar sites (TS).

Methods From November 2010 to December 2011, 300 patients undergoing various laparoscopic procedures were enrolled. Patient, surgery, and trocar characteristics were recorded. We evaluated at three time points (in-hospital and at 1 and 6 months postoperatively) specifically for each TS, pain (Visual Analog Scale), morbidity (infection, hematoma, hernia), and cosmesis (Patient Scar Assessment Score; PSAS). Patients designated their "worst TS," and a composite endpoint "bad TS" was defined to include any adverse outcome at a TS.

Results We analyzed 1,074 TS. Follow-up was $>90 \%$. Pain scores of $>3 / 10$ at 1 and 6 months postoperatively, were reported by 3 and $1 \%$ of patients at the $5 \mathrm{~mm}$ TS and by 9 and $1 \%$ at the larger TS, respectively ( $5 \mathrm{~mm}$ TS vs larger TS; $p=0.001)$. Pain was significantly lower for TS located in the lower abdomen than for the upper abdomen or the umbilicus $(p=0.001)$. The overall complication rate was $<1 \%$ and significantly lower for the $5 \mathrm{~mm}$ TS
\end{abstract}

A. Cristaudi $(\square) \cdot$ M.-L. Matthey-Gié $\cdot$ N. Demartines $(\square)$ Department of Visceral Surgery, Centre Hospitalier Universitaire Vaudois, 46 rue de Bugnon, 1011 Lausanne, Switzerland

e-mail: acristaudi@me.com

N. Demartines

e-mail: demartines@chuv.ch

D. Christoforidis

Department of Surgery, Ospedale di Lugano, Lugano, Switzerland (hematoma $p=0.046$; infection $p=0.0001$ ). No hernia was found. The overall PSAS score was low and significantly lower for the $5 \mathrm{~mm}$ TS $(p=0.0001)$. Significant predictors of "bad TS" were larger TS $(p=0.001)$, umbilical position $(p=0.0001)$, emergency surgery $(p=0.0001)$, accidental trocar exit $(p=0.022)$, fascia closure $(p=0.006)$, and specimen extraction site $(p=0.0001)$.

Conclusions Specific trocar morbidity is low and almost negligible for $5 \mathrm{~mm}$ trocars. The umbilicus appears to be an unfavorable TS.

\section{Introduction}

Laparoscopy has become the gold standard for a growing number of abdominal surgical procedures despite very few adequately powered prospective randomized studies. The further evolution of minimally invasive surgery includes single port laparoscopy (SPL) and natural orifice transluminal endoscopic surgery (NOTES).

The declared purposes of these even less invasive or "scarless" techniques is to further decrease morbidity and improve cosmetic results by reducing the number of incisions to a single surgical access or accessing the abdomen through natural orifices. Feasibility has been reported in several studies, but significant proof of benefit is still awaited. A few randomized controlled trials comparing SPL with multiport laparoscopy for various surgical procedures have been performed, showing longer operating times with a rate of conversion to multiport laparoscopy up to $51 \%$, and no difference in morbidity [1-6]. The cosmetic outcome, despite being frequently used as major argument to promote SPL, has been assessed only by few authors but without use of validated instruments [7, 8]. In 
many laparoscopic procedures, a mini-laparotomy is necessary to allow extraction of the surgical specimen. With SPL, all trocars are inserted through a port that is also used for specimen extraction. Therefore, the difference in "invasiveness" between multiport laparoscopy and SPL, at least in these cases, can only be derived from a decrease in morbidity specifically related to the additional trocars used in multiport laparoscopy. However, an exact quantitative analysis of the morbidity specifically related to TS is hardly available in the literature. There are some data on TS complications, such as infection and hernia [4-6], but they are mostly derived from retrospective studies without specific focus on the TS. The present study was therefore designed to assess prospectively all adverse outcomes specifically related to TS. It was also hypothesized that outcomes related to $5 \mathrm{~mm}$ trocar sites (5TS) are more favorable compared to larger trocar sites (LTS) and aimed at identifying other predictors of adverse outcome at the trocar site.

\section{Methods}

\section{Patients and data acquisition}

The present prospective observational study was performed in our tertiary care institution. It included all patients undergoing laparoscopic surgery with at least one $5 \mathrm{~mm}$ trocar. Patients undergoing pure SPL were not included. We also excluded patients if laparoscopy had to be converted to open surgery (unplanned necessity to perform a laparotomy longer than that needed for specimen extraction), if they were younger than 18 years or had language limitations, or if follow-up was impossible (patients operated on emergently in our department but living abroad). As this study was observational and did not involve any modification in the routine procedure, patients were asked to participate and signed an informed consent, usually on the day after surgery. Then, the investigators, together with the operating surgeon, filled out a detailed standardized data sheet (Appendix 1). Size, type, and precise trocar localization were recorded, together with patients' baseline characteristics, type and duration of surgery, and method of wound closure. Trocar sites replaced by a minilaparotomy for specimen extraction or a stoma were excluded from the analysis, as the present study focuse on trocars only. Location of TS was categorized as umbilical (in or around the umbilicus), upper abdominal (at or above the umbilical level), or lower abdominal. Trocars were categorized by size to $5 \mathrm{~mm}$ or LTS, including 10,12 , and $15 \mathrm{~mm}$. Single port access trocars were excluded from the study. The exact length of trocar incisions wasn't measured, because it was assumed that the operating surgeon tailored the incision according to the size of trocar used. The relationship between the trocar and length of incision was checked during follow-up.

Patient outcomes were assessed at three time points: shortly prior to discharge from the hospital but within 1 week of surgery (in-hospital assessment), at 1 month postoperatively, and at 6 months after surgery. In-hospital assessment was based on clinical examination in all patients. Evaluation of outcomes at 1 and 6 months was performed during outpatient visits, but patients who were unable or unwilling to attend were evaluated by a structured telephone interview. A study investigator (surgical resident or study nurse) assessed or asked the patient to report on the following outcomes, specifically for each trocar site: pain, surgical site infection (SSI), hematoma, hernia, cosmetic outcome, "worst TS," and "overall nuisance," based on a standardized questionnaire.

Pain was evaluated with the visual analogue scale (VAS) [9] at rest and with effort before discharge, and overall at 1 and 6 months. Surgical site infection was defined according to the Centers for Disease Control and Prevention criteria [10]. Hematoma was defined as bruising visible or palpable on an area more than $3 \mathrm{~cm}$ around the trocar site. Both were assessed prior to discharge and at 1 month. Hernia was assessed by clinical examination or by questioning for specific symptoms at 1 and 6 months. The cosmetic result was evaluated at 1 and 6 months with the validated "Patient Scar Assessment Score" (PSAS) [11], where the best possible score is 0 and the worst 60 (Appendix 2). We also asked patients at the three time points to indicate subjectively which trocar site they would have liked to avoid if the same surgery could have been performed with one trocar less ("worst TS"), and to rate all trocars on the VAS for "overall nuisance" (including pain, complications, or adverse cosmetic outcomes). Finally, we defined a composite endpoint "bad TS" as any trocar that fulfilled one or more of the following conditions: pain or overall nuisance rated with a VAS above $3 / 10$ at follow-up, elected as "worst TS," rated with a PSAS score above 6/60 or subject to a complication (SSI, hematoma, or hernia). Postoperative analgesia was not standardized but usually included paracetamol, metamizole, and opioids, according to hospital guidelines and based on surgery type and patient. Local anesthesia infiltration of trocar sites was not used.

Statistical analysis

Logistic regression analysis was performed to analyze the binary outcomes: SSI, hematoma, "worst TS," and "bad TS." To take into account the intragroup correlation (the observations are independent across groups/patients, but not necessarily within groups), the clustered sandwich 
Table 1 Laparoscopic procedures: patient and trocar distribution
$5 \mathrm{~mm}$ TS 5 millimter trocar site; LTS larger trocar site

\begin{tabular}{lllll}
\hline & Patients & $\begin{array}{l}\text { Number } \\
\text { of trocars }\end{array}$ & $5 \mathrm{~mm}$ TS & LTS \\
\hline Total & 300 & 1,074 & $477(44 \%)$ & $598(56 \%)$ \\
Emergency & $146(49 \%)$ & & & \\
Elective & $154(51 \%)$ & & $\mathrm{n} / 477$ & $\mathrm{n} / 598$ \\
& & & $109(23 \%)$ & $177(30 \%)$ \\
Cholecystectomy & 94 & $286(27 \%)$ & $148(23 \%)$ \\
Appendectomy & 80 & $247(23 \%)$ & $99(21 \%)$ & $147 \%)$ \\
Colon resection & 26 & $90(8 \%)$ & $47(10 \%)$ & $43(7 \%)$ \\
Rectal resection & 12 & $44(4 \%)$ & $21(4 \%)$ & $23(4 \%)$ \\
Rectopexy & 2 & $8(0.7 \%)$ & $4(0.8 \%)$ & $4(0.6 \%)$ \\
Gastric by-pass (or other bariatric procedure) & 42 & $241(22 \%)$ & $115(24 \%)$ & $126(21 \%)$ \\
Nephrectomy (hand-assisted) & 6 & $17(2 \%)$ & $6(1 \%)$ & $11(2 \%)$ \\
Surrenalectomy & 3 & $11(1 \%)$ & $2(0.4 \%)$ & $9(1 \%)$ \\
Splenopancreatectomy & 1 & $3(0.3 \%)$ & $2(0.4 \%)$ & $1(0.1 \%)$ \\
Nissen fundoplication & 6 & $30(3 \%)$ & $18(4 \%)$ & $12(2 \%)$ \\
Explorative laparoscopy (adhesiolysis, & 26 & $87(8 \%)$ & $49(10 \%)$ & $38(6 \%)$ \\
$\quad$ perforated ulcer suture, etc.) & & & & $3(0.6 \%)$ \\
Other & 2 & $4(0.8 \%)$ & $3(0.5 \%)$ \\
\hline
\end{tabular}

surgery; 1 patient died during surgery). Finally 300 patients were included, $154(51 \%)$ were operated electively and $146(49 \%)$ underwent an emergency procedure. Table 1 shows the type of procedures performed. There were 1,074 trocar sites on the 300 patients; 477 (44\%) were $5 \mathrm{~mm} \mathrm{TS}$, and $597(56 \%)$ were larger TS (LTS): 301 were $10 \mathrm{~mm}$ trocars, 256 were $12 \mathrm{~mm}$ trocars, and 40 were $15 \mathrm{~mm}$ trocars. All trocars were of the blunt tip type. Thirty-eight procedures were converted to minilaparotomy, and 9 required a stoma; these 47 operations were therefore excluded from the final analysis. Fascia closure was performed in $71 \%$ of LTS and $3 \%$ of $5 \mathrm{~mm}$ TS. The LTS in which the fascia was left open were mostly located below the xiphoid. In $60 \%$ of our patients, the resection specimen (appendix, gallbladder) was extracted through the umbilicus, and all umbilical trocars were LTS.

Follow-up

Patient follow-up was complete for all patients at the inhospital assessment, $90 \%$ at 1 month (87\% clinical assessment, $13 \%$ telephone interview), and $91 \%$ at 6 months $(59 \%$ clinical assessment, $41 \%$ telephone interview).

\section{Complications}

Complications related to the trocar sites are shown in Table 2. They were significantly less frequent in $5 \mathrm{~mm}$ TS compared to LTS. No trocar site hernia was found at the 6-month follow-up. 
Table 2 Trocar site morbidity

\begin{tabular}{llll}
\hline & $5 \mathrm{~mm}$ TS & LTS & $p$ Value \\
\hline Hematoma & $8(0.01 \%)$ & $71(6.6 \%)$ & 0.0001 \\
SSI & $1(0.001 \%)$ & $10(1 \%)$ & 0.046 \\
Hernia & 0 & 0 & \\
\hline
\end{tabular}

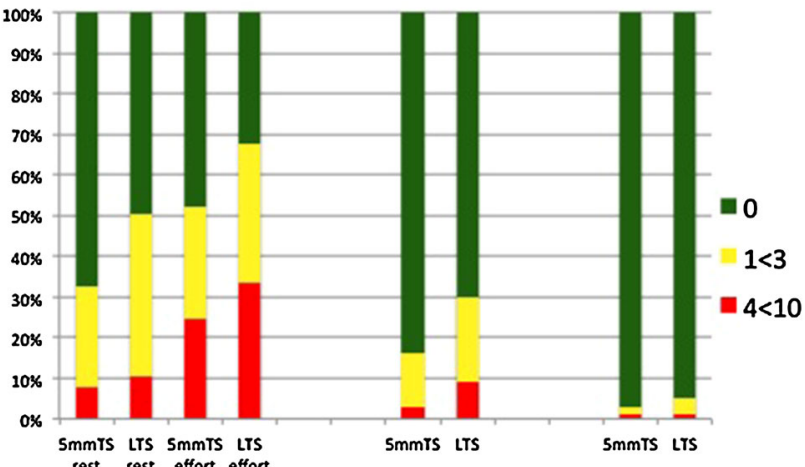

Fig. 1 Trocar site pain assessment. The Visual Analog Scale (VAS) score according to three main categories $(0 / 1-3 / 4-10)$ at the inhospital assessment (at rest and with effort), at 1 month, and at 6 months for $5 \mathrm{~mm}$ TS $5 \mathrm{~mm}$ trocar sites; LTS larger trocar sites (10, 12 , and $15 \mathrm{~mm}$ )

Pain

Pain at the TS was minimal for the majority of patients (Fig. 1). There were significant differences between $5 \mathrm{~mm}$ TS and LTS $(\mathrm{OR}=0.56, p=0.0001$, confidence interval [CI] $95 \%$ 0.47-0.67). At 1 month, patients reported pain of $3 / 10$ or less on the VAS scale in $97 \%$ for $5 \mathrm{mmTS}$ versus $91 \%$ for LTS, whereas at 6 months they reported pain of 3/10 or less in $99 \%$ of cases for both types of trocar site. Trocars placed in the lower abdomen caused significantly less pain $(\mathrm{OR}=0.57, p=0.001, \quad$ CI $95 \%$ $0.41-0.80)$.

"Overall nuisance"

Similarly, "overall nuisance" was rated $3 / 10$ or less on the VAS scale in $95 \%$ for $5 \mathrm{~mm}$ TS versus $88 \%$ for LTS. There were significant differences between $5 \mathrm{~mm}$ TS and LTS (OR 0.53, $p=0.0001,95 \%$ CI 0.39-0.73) (Fig. 2).

Cosmetic outcome

The cosmetic outcome as evaluated by the patient on the PSAS for each $5 \mathrm{~mm}$ TS was $6 / 60$ or less in $60 \%$ of patients at 1 month versus $41 \%$ for LTS. At 6 months, this proportion of patients increased to $80 \%$ for $5 \mathrm{~mm}$ TS

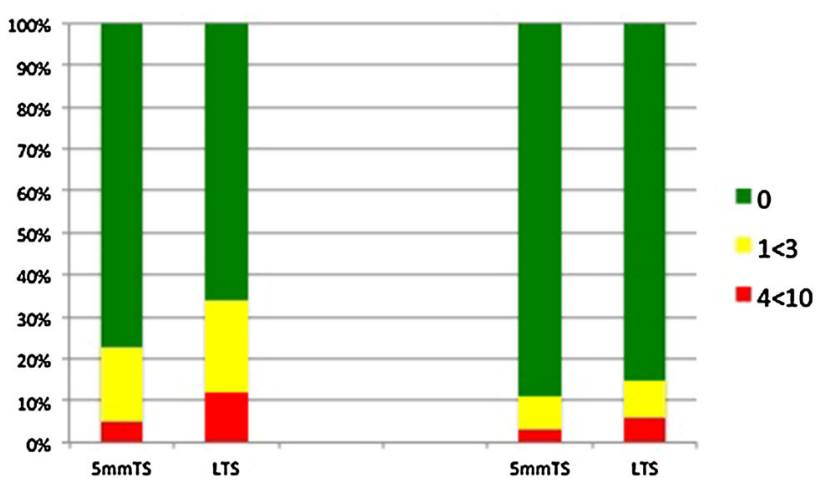

Fig. 2 Trocar site "overall nuisance" assessment (VAS). $5 \mathrm{~mm}$ TS vs LTS at 1 and 6 months postoperatively

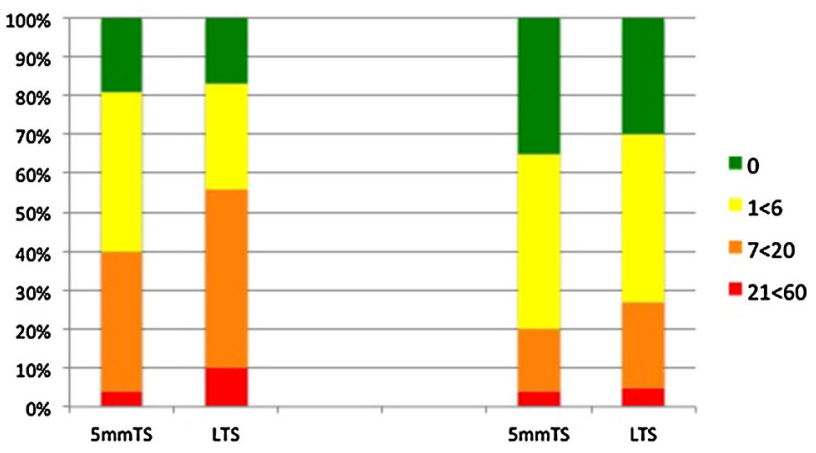

Fig. 3 Trocar site Patient Scar Assessment Score (PSAS). The PSAS was divided (arbitrarily) into four categories (0: no esthetical discomfort; $1<6$ : little discomfort; $7<20$ : mild discomfort; $21<60$ : big discomfort) for $5 \mathrm{~mm} \mathrm{TS}$ versus LTS at 1 and 6 months evaluation

versus $72 \%$ for LTS (Fig. 3). Patients were therefore significantly more satisfied with $5 \mathrm{~mm}$ TS scars than LTS scars (OR 0.57, $p=0.0001$, CI $95 \%$ 0.48-0.67).

\section{"Worst trocar"}

Five patients were unable to indicate a "worst TS," and 31 considered a TS converted to a minilaparotomy or stoma as "worst TS"; these patients were excluded from analysis. In the remaining patients, the "worst TS" was a LTS in $76 \%$ and a $5 \mathrm{~mm}$ TS in $24 \%$ (198 and 64 operations, respectively); 25 of these $5 \mathrm{~mm}$ TS had been used to exteriorize a drain. The difference between $5 \mathrm{~mm}$ TS and LTS was found to be statistically significant at univariate and multivariate analysis at the in-hospital assessment and at both 1 month and 6 months follow-up $(p=0.0001)$. The "worst TS" was most frequently located at the umbilicus (50\%), followed by the upper abdomen (31\%) and lower 
Table 3 Significant factors marking adverse outcomes for pain, "worst TS," overall nuisance, and PSAS

\begin{tabular}{|c|c|c|c|c|c|c|c|c|}
\hline \multirow[t]{2}{*}{ Outcomes } & \multicolumn{4}{|l|}{ Univariate analysis } & \multicolumn{4}{|l|}{ Multivariate analysis } \\
\hline & Factors & OR & $p(<0.05)$ & CI $95 \%$ & Factors & OR & $p(<0.05)$ & IC $95 \%$ \\
\hline \multirow[t]{8}{*}{ Pain (VAS) } & BMI & 0.96 & 0.0001 & $0.94-0.99$ & $5 \mathrm{~mm} \mathrm{TS}$ & 0.54 & 0.0001 & $0.43-0.67$ \\
\hline & $5 \mathrm{~mm} \mathrm{TS}$ & 0.56 & 0.0001 & $0.47-0.67$ & BMI & 0.96 & 0.001 & $0.94-0.99$ \\
\hline & Infra-umbilical location & 0.73 & 0.018 & $0.57-0.94$ & Infra-umbilical location & 0.57 & 0001 & $0.41-0.80$ \\
\hline & Emergency operation & 1.38 & 0.025 & $1.04-1.85$ & & & & \\
\hline & Umbilical location & 1.54 & 0.0001 & $1.29-1.84$ & & & & \\
\hline & Accidental exit & 1.57 & 0.002 & $1.17-2.09$ & & & & \\
\hline & Specimen extraction & 2.31 & 0.0001 & $1.87-2.85$ & & & & \\
\hline & Fascia closure & 1.95 & 0.0001 & $1.60-2.38$ & & & & \\
\hline \multirow[t]{7}{*}{ Worst incision } & Supra-umbilical location & 0.48 & 0.0001 & $0.33-0.71$ & $5 \mathrm{~mm} \mathrm{TS}$ & 0.15 & 0.0001 & $0.08-0.29$ \\
\hline & Infra-umbilical location & 0.32 & 0.0001 & $0.19-0.52$ & & & & \\
\hline & $5 \mathrm{~mm} \mathrm{TS}$ & 0.07 & 0.0001 & $0.04-0.13$ & & & & \\
\hline & Umbilical location & 5.63 & 0.0001 & $3.45-9.17$ & Accidental exit & 1.65 & 0.056 & $0.98-2.77$ \\
\hline & Accidental exit & 6.15 & 0.0001 & $3.32-11.4$ & Specimen extraction & 2.17 & 0.005 & $1.27-3.72$ \\
\hline & Specimen extraction & 956 & 0.0001 & $5.56-16.4$ & Fascia closure & 1.99 & 0.011 & $1.17-3.40$ \\
\hline & Fascia closure & 9.36 & 0.0001 & $5.76-15.21$ & Umbilical location & 1.77 & 0.004 & $1.19-2.62$ \\
\hline \multirow{5}{*}{$\begin{array}{l}\text { "Overall nuisance" } \\
\text { (VAS) }\end{array}$} & BMI & 0.97 & 0.049 & $0.93-0.99$ & BMI & 0.95 & 0.009 & $0.92-0.99$ \\
\hline & $5 \mathrm{~mm} \mathrm{TS}$ & 0.5 & 0.0001 & $0.39-0.66$ & $5 \mathrm{~mm} \mathrm{TS}$ & 0.53 & 0.0001 & $0.39-0.73$ \\
\hline & Umbilical location & 1.54 & 0.002 & $1.17-2.02$ & Specimen extraction & 1.5 & 0.037 & $1.02-2.18$ \\
\hline & Specimen extradion & 1.96 & 0.0001 & $1.41-2.73$ & & & & \\
\hline & Fascia closure & 1.65 & 0.002 & $1.21-2.25$ & & & & \\
\hline \multirow[t]{2}{*}{ PSAS } & Infra-umbilical location & 0.65 & 0.005 & $0.48-0.88$ & $5 \mathrm{~mm} \mathrm{TS}$ & 055 & 0.0001 & $0,45-0.68$ \\
\hline & $5 \mathrm{~mm} \mathrm{TS}$ & 0.57 & 0.0001 & $0.48-0.67$ & & & & \\
\hline cat 1 & & & & & & & & \\
\hline $1<6$ & Accidental exit & 2.12 & 0.0001 & $1.50-3.02$ & Accidental exit & 195 & 0001 & $1.29-2.94$ \\
\hline $7<20$ & Specimen extraction & 1.62 & 0.001 & $1.23-2.12$ & & & & \\
\hline \multirow[t]{2}{*}{$21<60$} & Fascia closure & 1.37 & 0.004 & $1.11-1.70$ & & & & \\
\hline & Umbilical location & 1.13 & non sign & & & & & \\
\hline
\end{tabular}

PSAS Patient Scar Assessment Score; VAS Visual Analog Scale; BMI body mass index

abdomen (19\%). This difference was statistically significant at the in-hospital assessment $(\mathrm{OR}=3.04$, $p=0.0001,95 \%$ CI 1.97-4.66), and still significant at 1 month and 6 months follow-up $(\mathrm{OR}=1.77, p=0.004$, $95 \%$ CI 1.19-2.62). Multivariate analysis confirmed that the umbilical location predicted a "worst TS" outcome, independent of size, fascia closure, and specimen extraction site (Table 3).

\section{Composite endpoint "bad TS"}

Three hundred seventy-one LTS (73\%) matched our definition of "adverse outcome" versus $1335 \mathrm{~mm}$ TS (27\%). We found that LTS, emergency surgery, intraumbilical localization, intraoperative accidental exit and reinsertion of trocars, specimen extraction site, and fascia closure were significant predictors of adverse outcome in univariate and multivariate analysis (Table 4).
Table 4 Predictors of "bad TS" (composite endpoint ${ }^{\mathrm{a}}$ )

\begin{tabular}{lll}
\hline & OR & $p$ Value \\
\hline Large TS & 3.22 & 0.001 \\
Umbilical location (vs upper or lower abdomen) & 3.17 & 0.0001 \\
Emergency operation & 1.54 & 0.0001 \\
Replaced trocar & 1.79 & 0.022 \\
Specimen extraction site & 2.21 & 0.001 \\
Fascia closure & 1.71 & 0.006 \\
\hline
\end{tabular}

${ }^{a}$ Defined as a trocar site where one or more of the following conditions were fulfilled: pain or overall nuisance rated with a VAS above 3 at follow-up, elected as "worst trocar," rated with a PSAS score above 6 or subject to a complication (surgical site infection [SSI], hematoma, or hernia)

\section{Patient factors}

Regarding patient-related factors (BMI, diabetes, oral anticoagulation, immunosuppression, tobacco use) we found a 
significant correlation between oral anticoagulation and postoperative hematoma $(\mathrm{OR}=3.37, p=0.001,95 \% \mathrm{CI}$ 1.67-6.81). Body mass index was inversely correlated with pain $(\mathrm{OR}=0.96, p=0.001,95 \%$ CI 0.94-0.99) and overall nuisance $(\mathrm{OR}=0.95, p=0.009,95 \%$ CI 0.92-0.99).

\section{Discussion}

To our knowledge, this study is the first objective and specific outcome analysis of laparoscopic trocars. We assessed prospectively the morbidity of over 1,000 trocar sites in 300 patients undergoing a multiport laparoscopic procedure. The present analysis provides detailed and specific quantitative assessment of the impact of individual laparoscopic trocars on the patient's well-being, pain, and cosmesis, using validated scores.

\section{Trocar size}

For $5 \mathrm{~mm}$ TS, the overall complication rate was $0.8 \%$, and relevant pain at 6 months was present in $1 \%$ of patients; a bad cosmetic result was reported in $4 \%$ of $5 \mathrm{~mm}$ TS. Larger trocar sites had a significantly worse outcome. This relationship between morbidity and trocar size may be an argument in favor of the development and use of even smaller (3 mm) laparoscopic trocars and instruments [13]. Interestingly, one randomized study found a benefit in term of operative length and postoperative pain with minilaparoscopic techniques compared to SPL [14].

\section{Cosmesis}

The cosmetic result from a patient's point of view, using various questionnaires and photos, after multiport laparoscopy vs SPL has been assessed by some authors but surprisingly not with validated scales or scores [12-19]. To our knowledge, our study is the first to analyze, from a patient's point of view, the cosmetic results with a validated score originally developed to assess patient satisfaction with the scar in plastic surgery [14]. Using this score, we showed a very low incidence of an adverse cosmetic outcome with $5 \mathrm{~mm}$ trocars. The only limitation of our cosmetic assessment lies in the fact that our study did not include a control group of patients without conventional TS, as for example patients undergoing SPL or NOTES.

\section{Morbidity}

Overall trocar-related morbidity was very low. Major bleeding at the $5 \mathrm{~mm}$ TS is rare [20], but spontaneously resolving minor bleeding and bruising occurs more frequently, although very few data are reported in the literature. We observed a rate of trocar site hematoma of $0.7 \%$ for $5 \mathrm{~mm}$ TS and $6.6 \%$ for LTS. Wound infections occurred only in one $5 \mathrm{~mm}$ TS and in 10 LTS; these results are in line with rates reported previously between $1 \%$ and $3 \%[3,4,21]$.

Swank et al., in a recent systematic review of trocar site hernia, reported a pooled prevalence between 0 and $5.2 \%$ in multiport laparoscopy. They also found a significant correlation with umbilical location and trocar size [20]. Studies comparing SPL with multiport laparoscopy report higher rates of incisional hernia for the larger SPL port sites (1.3-3.4\% for SPL versus $0.2-1.6 \%$ for multiport laparoscopy) $[1,3,4,22,23]$. In the present study, no trocar site hernias were diagnosed, but this may be related to the short follow-up limited to 6 months and the absence of systematic clinical and radiological assessment in $41 \%$ of patients, all asymptomatic at this time-point. To date, some 10 cases of trocar site hernia at a $5 \mathrm{~mm}$ TS have been reported in the literature, but we observed none in our study. General recommendations include fascial closure of every port $>10 \mathrm{~mm}$ and of those $<10 \mathrm{~mm}$ if prolonged manipulation occurs, but the time limit is not specified [23, 24]. In our study $3 \%$ of $5 \mathrm{~mm}$ trocar site fascia were closed because of enlargement of the fascial incision during prolonged manipulation.

\section{Localization of the trocar sites}

It is a common belief among surgeons that the umbilicus is an optimal site for trocar insertion, most likely because the scar can be hidden in the umbilical fold. Dauser et al. performed a preoperative survey of 150 patients planned for multiport laparoscopy. Patients gave significantly more importance to postoperative pain and return to normal activity than to cosmesis [16]. Bucher et al. performed a similar survey on 420 participants, including medical and paramedical staff, patients and general population [17]. Only $1 \%$ of responders put scars as a first postoperative priority. When participants were asked which surgical approach they would choose between multiport laparoscopy, NOTES, and SPL, assuming equal risk, SPL was the most common answer and the umbilicus the preferred access [18]. Our data challenge the idea that the umbilicus is the most ideal site for laparoscopic access. Not only was the umbilical site independently associated with more pain and a higher rate of SSI compared to other locations, but surprisingly, the cosmetic outcome was also rated worse by the patients, as reflected by the PSAS. Our assessment might be more realistic than hypothetical preoperative surveys as it represents the patient's point of view, registered postoperatively with a validated scale. In $60 \%$ of our patients, the resection specimen (appendix, gallbladder) was extracted through the umbilicus and all umbilical trocars were LTS. One can argue that this may have contributed to a worse outcome in terms of pain, morbidity, PSAS, and worst incision. In fact, size of trocar and site of specimen extraction 
were independent predictors of adverse trocar site outcome. However, in multivariate analysis the umbilical localization was a significant predictor of "bad TS," independent of trocar size and whether it had been used for specimen extraction or not. Our findings should alert surgeons to reconsider the idea of using the umbilicus as a port entry, in particular with SPL, where a larger incision is required.

Another interesting finding in our study is that lower abdomen TS cause significantly less pain than other locations, independent of size and other factors. A possible explanation may be the absence of the posterior rectus sheath in this area of the abdomen. This may have a clinical implication in those circumstances when the surgeon has the choice to place a trocar below or above the level of the umbilicus.

Finally, we found that the extraction (usually accidental) and re-insertion of trocars was independently associated with a worse outcome. This may be explained by enlargement of the fascial and peritoneal defect through the re-insertion maneuvers.

\section{Limitations}

Some limitations of this study need to be adressed. As stated in the Methods section, the follow-up was insufficient to detect all possible incisional hernias, especially in asymptomatic patients. The study population included 300 patients, which may appear to be a relatively small sample size, but our analysis was based on over 1,000 individual trocar sites with a standardized assessment protocol and use of validated scores. Outcome was assessed by an independent investigator or a study nurse, and only in rare cases, by the operating surgeon. Of note, the standardized follow-up questionnaire used was designed to allow for self-assessment by the patient for most of the outcomes, such as pain, cosmetic result, and overall nuisance. Postoperative analgesia was not standardized to allow a more objective pain assessment, but this proved to be impossible due to the different pathologies and types of laparoscopic procedures included. Analgesia however, was based on institutional guidelines.

Despite some limitations, the present study provides a specific and quantitative analysis of morbidity, pain, and cosmetic outcome related to individual trocar sites. Our results suggest that the overall negative impact of conventional trocar sites on patient well-being is very slight. Indirectly, they suggest that the margin of improvement in outcomes with SPL is very thin and that the threshold of adding a $5 \mathrm{~mm}$ trocar to facilitate SPL should be very low. Trocar morbidity depends on size and location of trocars, and therefore, we suggest that, when possible, smaller ( $5 \mathrm{~mm}$ or even $3 \mathrm{~mm}$ ) trocars should be used, preferably placed in the lower abdomen. The umbilicus as the preferred access point should be reconsidered, as it seems to be more painful and was poorly evaluated from a cosmetic point of view.

Acknowledgments The authors are grateful to Mrs. Mariotti for administrative help and to Dr. Faouzi for statistical analysis.

\section{Appendix 1}

Standardized Data Collection Questionnaire.
DONNEES PATIENT

\begin{tabular}{|c|c|c|c|c|c|}
\hline tools & \multicolumn{2}{|r|}{$\ln \rightarrow$} & $N$ & & ae \\
\hline nowats. & $\sim$ & $\infty$ & & & \\
\hline Tolater & $\sim$ & $\infty$ & meren & Goi & \\
\hline monestions & $\infty$ & $\infty$ & now: & & \\
\hline 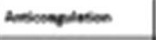 & ses & mat & 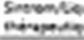 & & $\infty$ \\
\hline 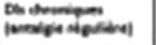 & $\sim$ & - & $\infty$ & & \\
\hline toraten ming & $\sim$ & -1 & $\infty$, & & \\
\hline Dopentes on & & & & & \\
\hline netse & & & $\cos 25$ & $=1$ & nesters \\
\hline nomen: & & & & & \\
\hline 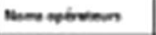 & & & & & \\
\hline pestipent & & ind & & & \\
\hline Comeriven & $\sim$ & - & Nomen: & & \\
\hline Comentive: & & & & & \\
\hline
\end{tabular}

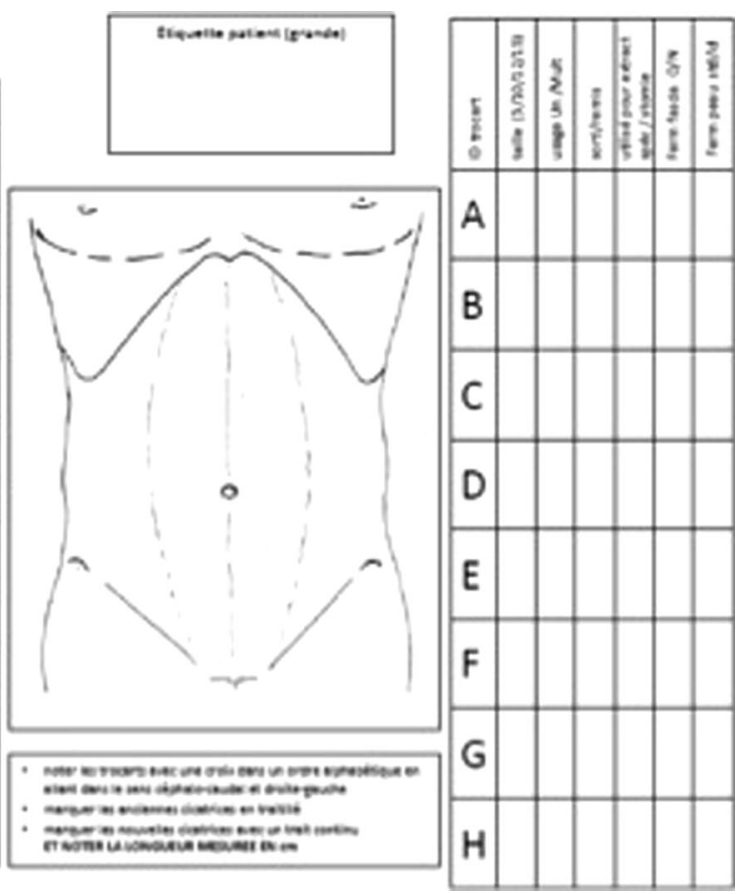




\section{Appendix 2}

Questionnaire: Patient Scar Satisfaction Score (PSAS) [11].

Circle the number corresponding to your opinion.

no complaints $\rightarrow$ worst imaginable

Is the scar painful?
Is the scar itching?

Is the colour of the scar different?

Is the scar more stiff?

Is the thickness of the scar different?

Is the scar irregular?

\begin{tabular}{cccccccccc}
1 & 2 & 3 & 4 & 5 & 6 & 7 & 8 & 9 & 10 \\
1 & 2 & 3 & 4 & 5 & 6 & 7 & 8 & 9 & 10 \\
normal skin & $\rightarrow$ & \multicolumn{1}{c}{ very different } \\
1 & 2 & 3 & 4 & 5 & 6 & 7 & 8 & 9 & 10 \\
1 & 2 & 3 & 4 & 5 & 6 & 7 & 8 & 9 & 10 \\
1 & 2 & 3 & 4 & 5 & 6 & 7 & 8 & 9 & 10 \\
1 & 2 & 3 & 4 & 5 & 6 & 7 & 8 & 9 & 10 \\
& & & & & & & Total: & 160
\end{tabular}

cultural adaptation, reliability evaluation and validation. Can J Surg 52:259-263

12. Dunker MS, Stigglebout AM, vaan Hogezand RA et al (1998) Cosmesis and body image after laparoscopic-assisted and open ileocolic resection for Crohn's disease. Surg Endosc 12:1334-1340

13. Saad S, Strassel V, Sauerland S et al (2013) Randomized clinical trial of single-port, minilaparoscopic and conventional cholecystectomy. Br J Surg 100:339-349

14. Fanfani F, Fagotti A, Gagliardi ML et al (2013) Minilaparoscopic versus single-port total hysterectomy: a randomized trial. J Minim Invasive Gynecol 20(2):192-197

15. Park SK, Olweny EL, Best SL et al (2011) Patient-reported body image and cosmesis outcomes following kidney surgery: comparison of laparoendoscopic single-site, laparoscopic and open surgery. Eur Urol 60:1097-1104

16. Dauser B, Görgel A, Stopfer J et al (2012) Conventional laparoscopy vs single port surgery from a patient's point of view: influence of demographics and body mass index. Wien Klin Wochenschr 124:834-841

17. Bucher P, Pugin F, Ostermann S et al (2011) Population perception of surgical safety and body image trauma: a plea for scareless surgery? Surg Endosc 25:408-415

18. Lamadé W, Friedrich C, Ulmer C et al (2011) Impact of body image on patient's attitude towards conventional, minimal invasive and natural orifice surgery. Langenbecks Arch Surg 396:331-336

19. Mohammadhosseini B, Shirani S (2011) Intra-abdominal and abdominal wall hematoma from $5 \mathrm{~mm}$ port insertion site in laparoscopic cholecystectomy. Videosurgery 6:164-166

20. Park J, Kwak H, Kim SG et al (2012) Single-port laparoscopic appendectomy: comparison with conventional laparoscopic appendectomy. J Laparoendosc Adv Surg Tech 22:142

21. Swank HA, Mulder IM, la Chapelle CF et al (2012) Systematic review of trocar-site hernia. Br J Surg 99:315-323

22. Yilmaz H, Alptekin H, Acar F et al (2013) Experiences of single incision cholecystectomy. Int J Med Sci 10:73-78

23. Yamamoto M, Minikel L, Zaritsky (2011) Laparoscopic 5-mm trocar site herniation and literature review. JSLS 15:122-126

24. Khurshid N, Chung M, Horrigan T et al (2012) 5-Millimeter trocar-site bowel herniation following laparoscopic surgery. JSLS $16: 306-310$ 